\title{
Ambulatory monitoring of patients with COVID-19: initial experiences and next steps
}

\author{
Stephen P Connolly (D) , ${ }^{1,2}$ Henriette Wa Katolo, ${ }^{1}$ Colm Cronin, ${ }^{1}$ \\ Alison Dingle, ${ }^{1}$ Maria Creed, ${ }^{1}$ Colin Edwards, ${ }^{3}$ Katherine M A O’Reilly, ${ }^{1,2}$ \\ Brendan O'Kelly, ${ }^{1}$ John Shearer Lambert, ${ }^{1,2}$ Eavan G Muldoon, ${ }^{1,2}$ \\ Gerard Sheehan, ${ }^{1}$ Heather Coetzee, ${ }^{1}$ Alan Sharp, ${ }^{1}$ Suzanne Dempsey, ${ }^{1}$ \\ Eileen O'Connor, ${ }^{1}$ Jeremy Farrell, ${ }^{1}$ Aoife G Cotter, ${ }^{1,2}$ Tara McGinty ${ }^{1,2}$
}

${ }^{1}$ Mater Misericordiae University Hospital, Dublin, Ireland ${ }^{2}$ University College Dublin School of Medicine, Dublin, Ireland

${ }^{3}$ PatientMpower Ltd, Dublin, Ireland

\section{Correspondence to} Dr Stephen P Connolly, Infectious Diseases, Mater Misericordiae University Hospital, Dublin, Ireland; sconnolly91@gmail.com

Received 1 November 2021 Accepted 11 February 2022

Check for updates

(C) Author(s) (or their employer(s)) 2022. No commercial re-use. See rights and permissions. Published by BMJ.

To cite: Connolly SP, Wa Katolo H, Cronin C, et al. BMJ Innov Epub ahead of print: [please include Day Month Year]. doi:10.1136/ bmjinnov-2021-000875

\section{INTRODUCTION}

Since cases were first described in December 2019, SARS-CoV-2 has posed a distinct challenge to healthcare delivery, and Ireland has been no exception. Hospital bed numbers per capita in Ireland are at 2.9 per 1000 inhabitants, bed occupancy is the highest in the European Union, ${ }^{1}$ care is predominantly delivered in 4 to 6-bedded wards, and single-room isolation facilities are in short supply, risking being overwhelmed by high caseloads. Droplet spread within environments increasingly appears to travel further than the initially predicted $2 \mathrm{~m}^{23}$ and one Irish hospital has reported as many as $49 \%$ of their COVID-19 cases occurring via nosocomial transmission, and higher (32\%) mortality in this group. ${ }^{4}$

We therefore identified a need to manage patients safely at home to minimise spread to susceptible patients and staff. As SARS-CoV-2 infection's natural history includes a rapid deterioration, characteristically in the second week of the illness in those who develop severe disease, ${ }^{56}$ the challenge of safely caring for such patients in the community was raised.

Given that COVID-19 causes pneumonitis and impaired oxygenation, it is advised that patients with mild-moderate COVID-19 are monitored for progression. $^{7}$ Finger probe oxygen saturation $\left(\mathrm{SpO}_{2}\right)$ monitoring is therefore a feasible method of home monitoring.

Within months of the pandemic being declared a number of centres internationally (including those within Australia, Canada, China, The Netherlands and

\section{Summary box}

What are the new findings?

- COVID-19 remote monitoring using oxygen saturation monitoring appears to be a safe and acceptable means of monitoring SARS-CoV-2-infected patients in the community for deterioration, reducing bed days spent in the hospital.

- Such application requires a highly protocolised service with a dedicated team and judicious application of the model to an appropriate subset of patients.

- In our cohort of 502 patients monitored remotely, 49 patients required repeat assessment in an acute hospital and 42 required admissions. Three patients required high dependency unit/intensive care unit admission and no patients died from COVID-19 complications.

How might it impact on healthcare in the future?

- A significant saving in bed days was observed by the service, with a potential net cost-benefit.

- This model may therefore be applied in future COVID-19 surges where bed shortages arise.

the UK) began to implement COVID-19 virtual monitoring programmes, taking a variety of forms, ranging from telephone support alone to remote assessments of patients' symptoms in combination with collecting biometric data. ${ }^{8}$ Ireland's national Health Service Executive (HSE) developed an early partnership with the digital health firm patientMpower in February 2020, with roll-out of a new 
COVID-19 monitoring service across many acute hospitals and general practice services across the country, with published positive experience ${ }^{9}$ Our institution was an early adopter of this service. Recent published international data (including a systematic review) highlights success of the model, however, individual studies using pulse oximetry as part of their protocol are limited in their number and sample size. ${ }^{8}$ We describe the largest single-centre cohort using oximetry found in the literature at the time of writing, detailing the planning and implementation for our COVID-19 Virtual Clinic (CVC), where patients with confirmed infection were monitored for deterioration in the community using a Bluetooth-enabled pulse oximeter and a bespoke mHealth (patientMpower) platform.

\section{METHOD}

Our project took place in Dublin's Mater Misericordiae University Hospital (MMUH), a busy inner-city tertiary hospital that is home to Ireland's National Isolation Unit. Our institution saw the initial cases of SARS-CoV-2 and has since treated a large proportion Dublin's hospitalised COVID-19 cases. Once community transmission became established by April 2020, the country's 'mitigation phase' commenced, and cases not requiring admission and with adequate means of home isolation were discharged to complete their isolation period in the community. Through close partnership with patientMpower and the hospital's COVID-19 steering committee, a CVC was established, with a team of redeployed staff from a variety of backgrounds, including the hospital's innovation department, nursing, audiology and physiotherapy. Staff were based in a clinic suite in the main hospital, with the option of home working where required. Each shift was overseen by a manager (usually infectious diseases experienced nurse specialist).

\section{CVC process}

From March 2020, both discharged inpatients deemed medically fit (afebrile for 48 hours, no oxygen requirement, able to self-isolate in appropriate accommodation) by their treating physicians, as well as ambulatory patients diagnosed by and discharged from the emergency department (ED) were referred to the COVID-19 CVC, given a Bluetooth enabled pulse oximeter (Nonin 3230; www.nonin.com) and asked to input two times a day readings of $\mathrm{SpO}_{2}$, heart rate and a self-reported dyspnoea score via a smartphone application (figure 1). Each patient was registered to a bespoke 'Digital Hub', allowing staff members to access the previous and new uploaded patient-recorded data. Patients were asked to upload biometric data at a minimum of two times a day (requested at 10:00 and 16:00) daily and in addition to abnormal readings, delays of longer than an hour prompted team members to call the patient. Patients typically required up to two

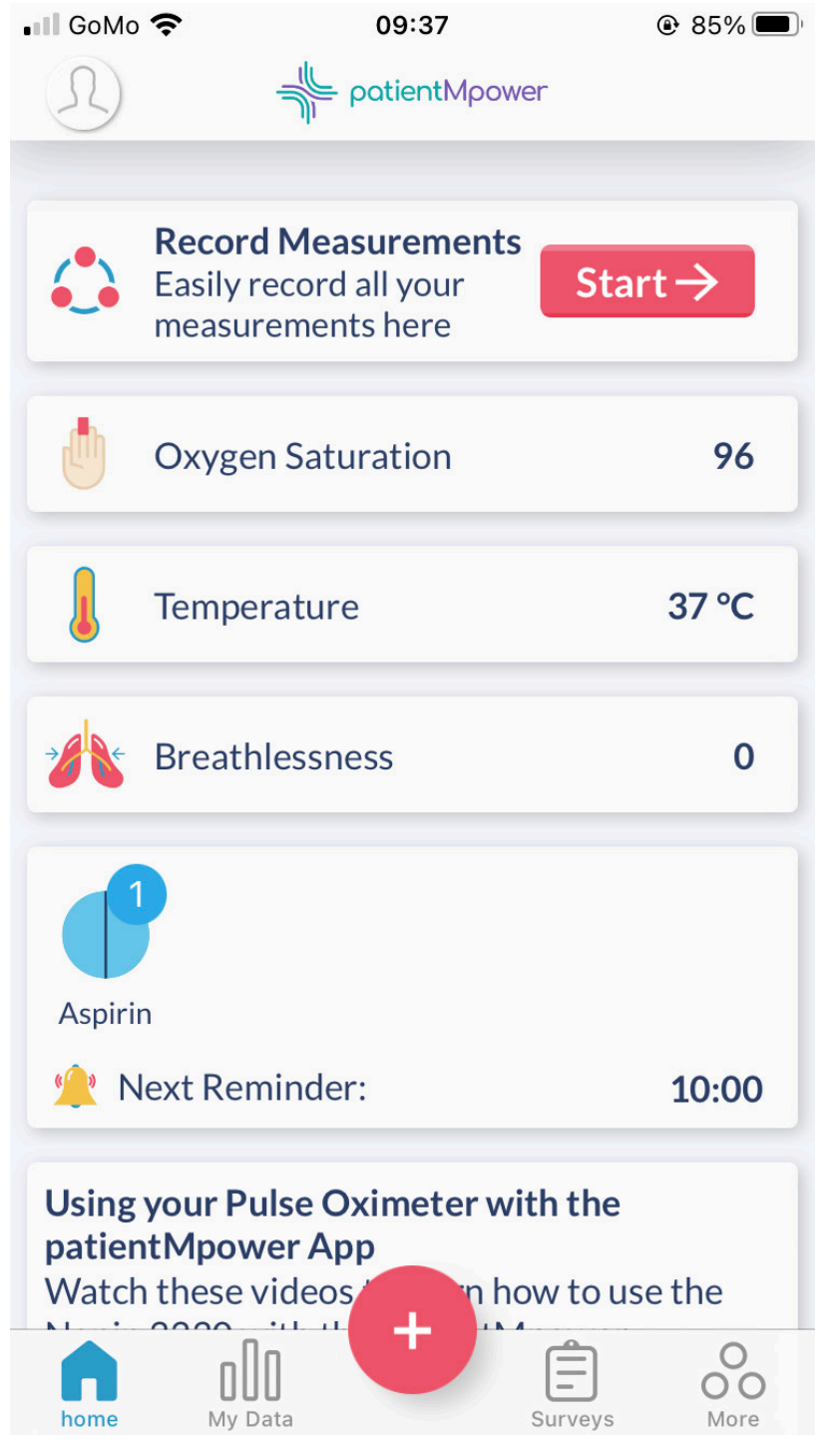

Figure 1 Image from patientMpower smartphone application. The interface allows users to input biometric data, with in-built video tutorials assisting in accurate data capture.

phone calls daily initially, with no further telephone calls required if data were within normal limits and uploaded regularly. Abnormal readings (ie, an $\mathrm{SpO}_{2}$ of $<94 \%$ or persistent tachycardia over 100 beats $/$ $\mathrm{min}$ ) triggered a notification to the virtual monitoring team, a call to the patient to review symptoms and assess the need for in-person review. All interactions were recorded on our institution's electronic medical records. For patients with persistently abnormal readings or concerning symptoms, an ambulance was called following consultation with the Infectious Diseases (ID) team to facilitate an urgent review during the day by the ID team or in-house on-call medical physician out of hours. A dedicated space in the ID unit was reserved for this purpose (figure 2). In some cases, following a telephone review from a member of the ID team, a patient's admission could be deferred, with more intensive frequency uploaded readings being requested, and regular calls to the patient where 


\section{Mater COVID Virtual Clinic under ID Team Governance}

\section{Monitoring Process}

\section{COVID Virtual Clinic (CVC)Team}

Patients registered on the Portal are:

$\checkmark$ Monitored twice daily, AM and PM

$\checkmark$ Phoned daily if problems using App or no daily measurements recorded

$\checkmark$ Ensure parameters are in normal range

- Oxygen saturation $\geq 94 \%$

- Temperature $<37.5^{\circ} \mathrm{C}$

- Breathlessness Scale < 4

\section{If parameters abnormal phone Patient} to re-submit readings

If still abnormal escalate to NIU Consultant / ID Reg or 8pm-8am COVID Reg on call, Bleep

If reassessment / admission required refer to: 'Community to Covid-19 Cohort Area Algorithm'

Document all patient interactions in 'COVID Remote Monitoring' document on PatientCentre

Monitoring complete 14 days post date of first symptom or 14 days post swab result, in absence of alternate guidance from ID consultant

$\checkmark$ Call patient for final check

$\checkmark$ If well, inform that monitoring is complete $\&$ device will be collected

$\checkmark$ Staff members advised to email (occupational health) if queries on retum to work process

\begin{tabular}{l} 
- Archive patient on patientMpower \\
portal; this auto generates email \\
advising that courier will collect device \\
- Document monitoring completion in \\
'COVID Remote Monitoring' \\
document on PatientCentre \\
- Remove patient from CoVID Remote \\
Worklist on PatientCentre \& status as \\
'Remote Monitoring Complete' \\
\hline \\
- Courier arranges device collection \\
- Device \& decontaminated for re-use \\
\hline - Pulse oximeters returned to MMUH for \\
re-use
\end{tabular}

\section{CVC Coordinated Re-Assessment Process}
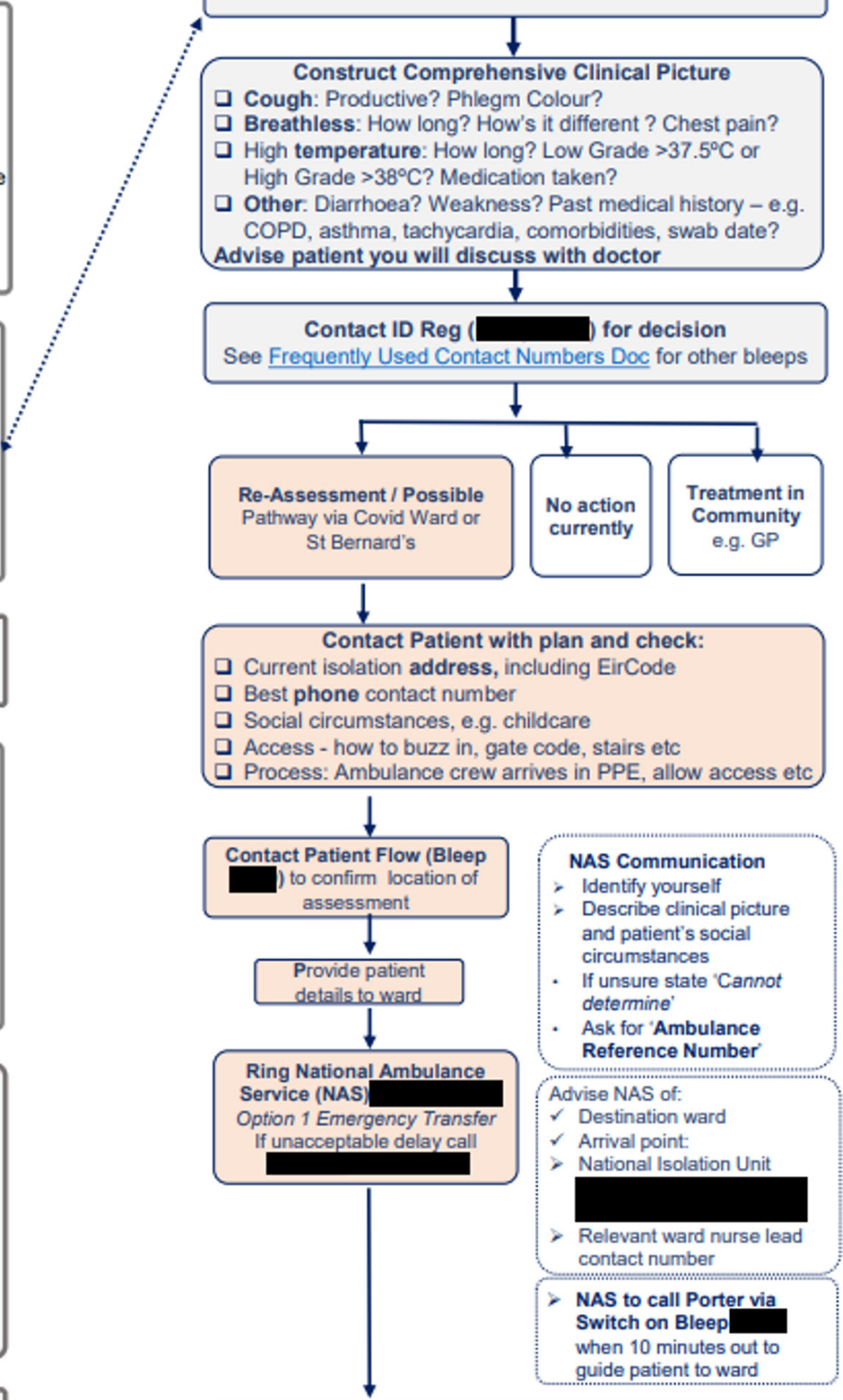

- Document in 'COVID Remote Monitoring' on PatientCentre - Change COVID Remote Worklist Status

Out of hours: Patient rings COVID Reg on call via Switch Reg follows Community to COVID-19 Cohort Area Algorithm

Figure 2 Monitoring and escalation process, CVC (V.4.0, dated 05 June 2021). Specific dates and contact numbers have been removed. ID team, Infectious Diseases team. Abbreviations/acronyms used: COPD, chronic obstructive pulmonary disease; GP, general practitioner; ID, infectious diseases; NIU, national isolation unit; PPE, personal protective equipment; Reg, registrar. 
appropriate. Patients were typically monitored for a total of 14 days following the onset of symptoms, with patients having the option of remaining on the service at clinician discretion.

\section{Evaluation process}

To assess this mode of care, our team retrospectively collected anonymised data on patients referred to the clinic between 1 March and 1 June 2020. Records were interrogated to find a complete list of CVC patients who had been using the service who subsequently presented to the hospital, including unscheduled presentations to the ED. Data on baseline demographics and reason for re-assessment were collected from charts, online medical records and the patientMpower online portal. Routine calls, such as initiation or conclusion of monitoring were excluded.

An analysis was also performed of bed days and costs saved during the study period. Given the large number of healthcare workers monitored that may not otherwise have been referred to MMUH, this analysis excluded occupational referrals not requiring admission or readmission. Median length of stay for patients without use of the CVC service, outpatients admitted onto the CVC service and inpatients discharged on to the CVC service were calculated to compare bed utilisation. Given the lack of data on proportions of those presenting to the ED with COVID-19 symptoms that would have otherwise been admitted for observation had the CVC service not existed; a sensitivity analysis was performed using a selection of proportions. Costsaving analysis was performed using the number of saved bed days calculated and national bed occupancy costs, and offset against average salaries of those redeployed to the service and additional running costs.

Descriptive statistics were employed to describe the patient characteristics of those enrolled in the CVC. Univariate regression was employed to determine factors associated with likelihood of requiring subsequent reassessment. All analysis was carried out using IBM SPSS Statistics for Windows, V.26. Data are presented as $\mathrm{n}(\%)$ or median (IQR) unless otherwise stated. Missing data values were excluded from the analysis.

\section{RESULTS}

Five hundred and two patients had initiated and completed monitoring between 1 March and 1 June 2020. Median time on the service was 12 (IQR 13-10) days. Median age was 40 (IQR 50-30) years. $63.3 \%$ were female, and $73.2 \%$ were healthcare workers.

\section{Calls to and from the service}

Thirty-nine patients made calls to the monitoring service, with most calling on a single occasion and only three patients calling two times. Typical documented reasons for calls included symptoms other than dyspnoea (53.8\%), and non-clinical queries (including technical issues with software; 17.5\%). In total, 1902 calls from the monitoring service to 442 patients ( $88 \%$ of the cohort) were documented, constituting a median of three calls per patient (IQR 1-5 calls). The most frequent reasons for calls to patients were absent readings (occurring with $50.1 \%$ of patients receiving calls), and abnormal $\mathrm{SpO}_{2}(30.1 \%)$ and technical issues (most commonly delays with pulse oximeter delivery and device pairing issues with mobile phones; almost $20 \%)$. Technical issues were typically resolved with telephone guidance.

\section{Patient outcomes}

A total of 49 patients $(9.8 \%)$ presented acutely for assessment (48 (98.0\%) to MMUH and 1 (2\%) to another hospital) and 42 of the $48(87.5 \%)$ presenting to our hospital were readmitted. Three patients had diagnoses unrelated to COVID-19 and one further patient was readmitted due to problems relating to self-isolation. Of the remaining 45 patients included in the analysis, $33(73.3 \%)$ were advised to present for assessment by the COVID-19 CVC, the remainder opting instead to present directly to the ED instead. The reasons for presenting in the cohort who presented to the ED without contacting the CVC were chest pain ( 2 patients, 16.7\%), dyspnoea (4 patients, $33.3 \%$ ) and low observed $\mathrm{SpO}_{2}$ readings (6 patients, $50.0 \%)$. The median number of days after enrolling on the monitoring service that patients typically presented for assessment was 5.0 days (IQR 8.0-3.0, data missing for one patient). Of those re-presenting for assessment, dyspnoea was the most common presenting complaint $(24,53.3 \%) ; 19(42.2 \%)$ had low $\mathrm{SpO}_{2}$ without dyspnoea and all the high dependency unit/intensive care unit admissions were from this latter group. Median length of stay was 4.42 days (IQR 1.0-6.5) in the readmitted group overall. Three patients $(6.7 \%)$ required critical care admissions and none died. A single patient of the cohort has died since monitoring, the cause of death relating to a preexisting terminal diagnosis. Other than demographics, data of the patient who presented to another hospital are absent.

Cost-saving analysis

The median length of stay for COVID-19 cases in the hospital during the study was 8 days (IQR 3-17). This was applied to the bed days saved for these cases. Of those admitted to the hospital from this cohort, the median length of stay was 6 (IQR 2-9) days, 2 days fewer than those not on remote monitoring. Therefore 2 days length of stay was applied to these cases in subsequent calculations. The cost savings per bed days saved was calculated using Ireland's national multioccupancy room rate of $€ 813$ per night. ${ }^{10}$ The costs of the system and equipment were provided free-ofcharge to the hospital and financially supported by the 
Ireland's HSE, leaving staff wages as the only running cost of the service. The gross average annual salary of the redeployed healthcare workers (HCWs) to the CVC was estimated at $€ 60000$, or a monthly salary of $€ 5000$. ${ }^{11}$ The total gross salary paid to CVC during the intervention was therefore $€ 270000$. These costs, minus the savings from the bed days avoided, estimates a range of results ranging from a net cost to the health service of over $€ 142000$ (assuming only $10 \%$ of patients discharged to the CVC would otherwise have been admitted), to net savings of $€ 27883$ (assuming a 30\% admission rate) to $€ 198288$ (assuming 50\%\% admitted). An admission rate of $27 \%$ was required to demonstrate a net cost-benefit.

\section{DISCUSSION}

The MMUH COVID-19 CVC/home monitoring service represents one of the largest cohort of home monitored COVID-19 patients using home $\mathrm{SpO}_{2}$ monitoring. This system requires judicious application to low-risk patients, a trained team, and a highly protocolised service with access to specialist rapid inpatient assessment. Our experience highlights the value of dynamic monitoring of patients which allows the prompt identification of hypoxia, which is $52.6 \%$ occurred in the absence of symptoms.

While we feel this system has worked well in our institution's response to COVID-19, reducing the cohort of hospitalised patients with COVID-19; the service, and indeed this evaluation of it, is not without issue. Several patients presenting for assessment with low $\mathrm{SpO}_{2}$ had normal saturations on formal assessment suggesting either transient hypoxia or pulse oximeter issues. The high proportion of patients who were healthcare workers in this cohort questions the generalisability to other populations. Regarding the cohort of patients who presented directly to the hospital or phoned for medical assistance, data are not collected with respect to the reason for this. Potential reasons may include unfamiliarity with the escalation process or presence of out-of-hours support, or potentially difficulties being connected to the medical team on call outside of the CVC's staffed hours. While this service aims to identify deteriorating patients in the community, it anecdotally has had success in reassuring dyspnoeic patients with normal recorded oxygen saturations and thus preventing unnecessary presentations for assessment. Scope therefore exists for further qualitative studies. Given the regular telephone support available to all patients on the service, the benefit that pulse oximetry individually confers to mortality or presentation rates is unclear. The true number of bed days saved by the intervention is also unclear given difficulties in predicting likelihood of admission without home monitoring. While we calculate that an admission rate of just over $26 \%$ was required for net cost-effectiveness, the bed days saved and home isolation alone arguably justify the intervention alone. The avoidance of ED time that the service allowed is an additional, unquantified, saving. On-going challenges to providing this service in future waves include the issue of staffing; as 'non-COVID' care resumes, redeployed staff are rapidly required to fill their original role. Dedicated staffing is key to maintaining continuity in our experience. Our resolving staff base in the prevaccination period however included many for whom direct patient contact was contraindicated due to health concerns, and this is a viable and useful option to use their clinical expertise effectively.

A large systematic review published in The Lancet this year assessed a variety of similar models. The authors acknowledge the heterogeneity of these interventions and the impact on the conclusions that can be drawn, as well as a need for greater emphasis on patient satisfaction measures. They also point out difficulties in assessing economic benefit, sustainability (especially where staffing is concerned) ${ }^{8}$

Another, more recent publication matched patients with suspected COVID-19 discharged from the ED symptoms and discharged with remote monitoring to a cohort of patients discharged without such follow-up. ${ }^{12}$ They found a need for reassessment in $69 \%$ of monitored patients compared with $85 \%$ in the control group $(\mathrm{p}=0.023)$, as well as a $58 \%$ reduction in bed occupancy.

Our experience describes one of the largest remotely monitored cohorts of COVID-19 patients published to date. The low frequency of readmissions and the usefulness of $\mathrm{SpO}_{2}$ monitoring and dyspnoea scores in predicting and recognising the need for readmission highlights the value of the CVC model in providing safe and potentially cost-effective remote care to those with COVID-19 as we continue to find innovative solutions to the problems posed by the pandemic.

Twitter Colin Edwards @ColinEdwards767

Acknowledgements The authors would like to sincerely thank those who so rapidly established and maintained this service, as well as patientMpower for their support. Specifically, we wish to thank our steering committee, Suzanne Draper, Sean Paul Teeling, Roulla Katiri, Nina Sivan, Michelle McGuirk, Julie Wynne, Katie Halsted, Marie Brid Casey, Meave Keaveney, Irene Byrne, Caroline Walsh, Jessica Kenny, Michelle Mullen, Aileen Gill, Kieran McDonnell and the National College of Art and Design.

Contributors Each author made substantive contribution to the design of the service and study, the data collection, data analysis or manuscript authorship. Each was sent a copy of each iteration of the manuscript for approval and suggested edits. The study and service were designed and steered by TM, AGC, JSL, GS, AS, SD, KMAO'R, MC and HC. The intervention was run and operated by MC, EO'C and JF. Data collection was undertaken by SPC, CC and HWK. Data analysis was performed by SPC. Manuscript writing was completed by SPC, with input from B'OK and $\mathrm{AD}$. CE is a member of the patientMpower scientific team who reviewed the manuscript. The remote monitoring programme was developed by patientMpower Ltd with advice from external clinical experts (C. O'Seaghdha, and R. Costello of Beaumont Hospital, and KMAO'R of the Mater Misericordiae University Hospital). The deployment of the remote monitoring programme free of charge to clinical care centres in Ireland was supported by the Health Service Executive HSE of Ireland. 
Funding The authors have not declared a specific grant for this research from any funding agency in the public, commercial or not-for-profit sectors.

Competing interests The only authors with conflicting interests are as follows: $\mathrm{CE}$ is an employee of $\mathrm{pMp}$, the private company which devised the smartphone application used in the intervention. KMAO'R has provided expert consultation to $\mathrm{pMp}$ in the past.

Patient consent for publication Consent obtained directly from patient(s).

Ethics approval This study involves human participants and was approved by the MMUH Research Ethics Committee (8 April 2020; reference 1/3782141). Express written consent was obtained from one patient to describe the events leading to her admission in detail. Ethical approval was sought to collect and analyse patient information in anonymised, aggregate form.

Provenance and peer review Not commissioned; externally peer reviewed.

ORCID iD

Stephen P Connolly http://orcid.org/0000-0002-7814-1167

\section{REFERENCES}

1 OECD. OECD/European Observatory on health systems and policies (2019), ireland: country health profile 2019, state of health in the EU. Brussels: OECD publishing, Paris/European Observatory on health systems and policies, 2019.

2 Setti L, Passarini F, De Gennaro G, et al. Airborne transmission route of COVID-19: why 2 meters/ 6 feet of inter-personal distance could not be enough. Int J Environ Res Public Health 2020;17:2932.

3 Bahl P, Doolan C, se Silva C. Airborne or droplet precautions for healthcare workers treating COVID-19. J Infect Dis 2020;16:jiaa189.

4 Reidy N. Royal College of Physicians Ireland Online Lecture Series: COVID-19 - COVID-19: Lessons from Wave One [Internet], 2021. Available: https://courses.rcpi.ie/product?
catalog=COVID-19-A-Clinical-Perspective-online-recording [Accessed 18 May 2021].

5 Guan W-jie, Ni Z-yi, Hu Y, et al. Clinical characteristics of coronavirus disease 2019 in China. N Engl J Med Overseas Ed 2020;382:1708-20.

6 Zhou F, Yu T, Du R, et al. Clinical course and risk factors for mortality of adult inpatients with COVID-19 in Wuhan, China: a retrospective cohort study. Lancet 2020;395:105462.

7 Gandhi RT, Lynch JB, del Rio C. Mild or moderate Covid-19. N Engl J Med Overseas Ed 2020;383:1757-66.

8 Vindrola-Padros C, Singh KE, Sidhu MS, et al. Remote home monitoring (virtual wards) for confirmed or suspected COVID-19 patients: a rapid systematic review. EClinicalMedicine 2021;37:100965.

9 Edwards C, Costello E, Curley M. Audit of the Covid-19 remote patient management programme in Ireland. presented at Irish thoracic Society annual scientific meeting, 2020. Available: https://www.ncbi.nlm.nih.gov/pmc/articles/ PMC7788179/

10 Hospital Charges [Internet], 2021. Available: https://www.hse. ie/eng/about/who/acute-hospitals-division/patient-care/hospitalcharges/ [Accessed 17 May 2021].

11 Consolidated Salary Scales in Accordance With Fempi 2015 And The Public Service Stability Agreements 2013 - 2020 (The Lansdowne Road Agreements [Internet]. Hse.ie, 2020. Available: https://www.hse.ie/eng/staff/resources/hr-circulars/ 1-january-2020-consolidated-payscales.pdf [Accessed 5 Apr 2021].

12 Dirikgil E, Roos R, Groeneveld GH, et al. Home monitoring reduced short stay admissions in suspected COVID-19 patients: COVID-box project. Eur Respir J 2021;58. doi:10.1183/13993003.00636-2021. [Epub ahead of print: 05 08 2021]. 